\title{
A study on the probabilistic response of typical sound insulation systems inside and outside room with arbitrary random excitation
}

\author{
Mitsuo Ohta* and Yasuo Mitani** \\ *Faculty of Engineering, Hiroshima University, \\ Shitami, Saijo-cho, Higashi-Hiroshima, 724 Japan \\ **Faculty of Engineering, Fukuyama University, \\ Sanzo, Higashimura-cho, Fukuyama, 729-02 Japan
}

(Received 18 October 1985)

\begin{abstract}
In this report, when a general stationary random noise of non-Gaussian arbitrary distribution type is attenuated by various kinds of sound insulation systems, such as a singlewall, a double-wall and a barrier, a simplified probabilistic evaluation method for the output random noise fluctuation is theoretically proposed in a unified form of the probability distribution expression. The validity of the proposed evaluation method is experimentally confirmed by applying it to the actual noise data.
\end{abstract}

PACS number: 43. 60. Cg, 43. 55. Jz

\section{INTRODUCTION}

As is well-known, the sound insulation wall and barrier are very often constructed as a typical noise control system. The acoustical design and/or evaluation problems of walls and barriers have been already considered by many investigators. ${ }^{1-7)}$ Almost all of these studies were confined only to the deterministic or average evaluation of the shielding effect. In the actual noise environment, however, the noise fluctuation emitted from sound sources shows very often an irregular time pattern with intricate ups and downs, and various kinds of probability distribution forms apart from a standard Gaussian distribution form. Furthermore, the statistics such as a median, $L_{x}$ sound levels and $L_{\text {eq }}$ evaluation index are very important for the actual noise evaluation and regulation problems. In this case, it is necessary to establish an explicit expression of the output noise level or noise intensity distribution function in close relation to the frequency spectral characteristic of the input noise fluctuation and the sound insulation system.

From the methodological point of view, there are two ways of approaches for predicting the above probability distribution. As one method, especially from the relative viewpoint of the sound characteristic change of a sound insulation system, some prediction method of this kind has been proposed in Ref. 8). On the other hand, another method is the direct method of evaluating the resultant probability distribution due to the new construction of a sound insulation system, not from the relative viewpoint due to the above system change. Based on the latter point of view, in this report, a simplified and unified statistical evaluation method for predicting the output noise level (or noise intensity) probability distribution is theoretically proposed. The validity of the proposed evaluation method is experimentally confirmed by applying it to the actual noise data.

\section{THEORETICAL CONSIDERATION}

2.1 General Expression for Noise Intensity Distribution

In order to predict the noise evaluation index, $L_{x}$, it is first necessary to find a unified explicit expression of the probability density function (abbr., p.d.f.) 
with respect to the output noise level or noise intensity fluctuations. As was reported previously, the generally applicable expression on the above p.d.f. is a series expansion type expression taking a gamma distribution function as the first expansion term. More concretely, paying special attention to the fact that the noise intensity, $E$, fluctuates always in a nonnegative region $[0, \infty)$, the probability density function $P(E)$ can be generally expressed in the general form of a statistical Laguerre expansion series ${ }^{9)}$ :

$$
P(E)=P_{\Gamma}(E ; m, S)\left[1+\sum_{n=3}^{\infty} A_{n} L_{n}^{(m-1)}\left(\frac{E}{S}\right)\right]
$$

with

$$
\begin{gathered}
m=\frac{\langle E\rangle^{2}}{\left\langle(E-\langle E\rangle)^{2}\right\rangle}, \\
S=\frac{\left\langle(E-\langle E\rangle)^{2}\right\rangle}{\langle E\rangle}, \\
A_{n} \triangleq \frac{\Gamma(m) n !}{\Gamma(m+n)}\left\langle L_{n}^{(m-1)}\left(\frac{E}{S}\right)\right\rangle \\
=\sum_{K=0}^{n} \frac{(-1)^{K}}{K !} \frac{\Gamma(m) n !}{\Gamma(n-K+1) \Gamma(m+K)} \frac{\left\langle E^{K}\right\rangle}{S^{K}}
\end{gathered}
$$

and

$$
P_{\Gamma}(E ; m, S)=\frac{1}{\Gamma(m) S^{m}} e^{-E / S} E^{m-1}
$$

(gamma distribution function).

Hereupon, $L_{n}^{(m-1)}(\cdot)$ and $\langle *\rangle$ denote the associated Laguerre's polynomial and an averaging operation with respect to $*$. At this time, the cumulative distribution function (abbr., c.d.f.), $Q(E)\left(\triangleq \int_{0}^{E} P(\xi) d \xi\right)$, for the noise intensity fluctuation, which is very important for the purpose of finding any $L_{x}$ sound level, is expressed as follows ${ }^{9)}$ :

$$
\begin{aligned}
Q(E)= & \int_{0}^{E / S} P_{\Gamma}(\xi ; m) d \xi \\
& +P_{\Gamma}\left(\frac{E}{S} ; m+1\right) \sum_{n=3}^{\infty} B_{n} L_{n-1}^{(m)}\left(\frac{E}{S}\right)
\end{aligned}
$$

with

$$
P_{\Gamma}(\xi ; m) \triangleq \frac{1}{\Gamma(m)} e^{-\xi} \xi^{m-1}
$$

(standard gamma distribution)

and

$$
B_{n} \triangleq \frac{(n-1) ! \Gamma(m+1)}{\Gamma(m+n)}\left\langle L_{n}^{(m-1)}\left(\frac{E}{S}\right)\right\rangle
$$

$$
=\sum_{K=0}^{n} \frac{(-1)^{K}}{K !} \frac{(n-1) ! \Gamma(m+1)}{\Gamma(n-K+1) \Gamma(m+K)} \frac{\left\langle E^{K}\right\rangle}{S^{K}} .
$$

2.2 Relationship between Frequency Spectral Characteristic of Sound Insulation System and Parameters Contained in C.D.F. Expression

Now, let $X_{i}(i=1,2, \ldots, N)$ be an input noise intensity fluctuation existing in the $i$ th frequency bandwidth of the noise fluctuation wave emitted from a sound source. And let the transfer coefficient $a_{i}$ $(i=1,2, \ldots, N)$ reflect the frequency spectral characteristic of the sound insulation system at the center frequency, $f_{\mathrm{c} i}$, of the $i$ th octave band (or one-thirdoctave band). From the additive property of noise intensity, an over-all noise intensity, $E$, attenuated by the sound insulation system at an observation point is obviously given by:

$$
E=\sum_{i=1}^{N} a_{i} X_{i} .
$$

Thus, based on the information on the statistical property of $X_{i}$ and the frequency spectral characteristic $a_{i}(i=1,2, \ldots, N)$, the $p$ th order moment, $\left\langle E^{p}\right\rangle$, for $E$ can be easily estimated as follows:

$$
\left\langle E^{p}\right\rangle=\sum_{i+j+\ldots+n=p} \frac{p !}{i ! j ! \ldots n !} a_{1}^{i} a_{2}^{j} \ldots a_{N}^{n}\left\langle X_{1}^{i} X_{2}^{j} \ldots X_{N}^{n}\right\rangle \text {. }
$$

Therefore, based on these lower and higher order moments, many values of parameters $m, S$ and $B_{n}$ can be calculated by use of Eqs. (2) and (7). Then, the c.d.f. of the output noise intensity fluctuation can be evaluated by use of Eq. (5). The effect of the frequency spectral characteristic and the moment statistics of the incident noise on the objective c.d.f. expression is explicitly reflected in each parameter $m, S$ and $B_{n}$.

\subsection{Evaluation of Frequency Spectral Character-} istic for Sound Insulation System

In this section, let us estimate the frequency spectral characteristic for single- and double-walls as the sound insulation system inside a room and the barrier as the sound insulation system outside a room.

2.3.1 Frequency spectral characteristic of sound insulation wall

As one of the typical methods for evaluating the frequency spectral characteristic, $a_{i}(i=1,2, \ldots, N)$, of a sound insulation wall, it is possible to employ the well-known statistical energy analysis method 


\section{OHTA and Y. MITANI: PROBABILISTIC RESPONSE OF SOUND INSULATION SYSTEMS}

(S.E.A. method) by Crocker and Price (i.e., see Ref. 4) for the single-wall case and Ref. 5) for the doublewall case). For the double-wall case, however, considerable discrepancies between the theoretically estimated value by use of the Price and Crocker's method $^{5)}$ and the experimentally observed value can be seen, especially in the low frequency region. For the purpose of achieving the good agreement in the above low frequency region, the improved S.E.A. method for the double-wall case was proposed after introducing a different proper power flow. ${ }^{10)}$ In the previous paper, ${ }^{8)}$ its concrete evaluation method of the frequency spectral characteristics for each wall is shown by use of the above two S.E.A. methods.

2.3.2 Frequency spectral characteristic of sound insulation barrier

Now, let us consider a sound insulation barrier shown in Fig. 1. Assuming that the fixed sound source can be regarded as a point source and the barrier is sufficiently long in the horizontal direction, the Fresnel number, $N_{i}$, at the center frequency, $f_{\text {ci }}$, of the $i$ th octave band (or one-third-octave band) can be firstly determined as:

$$
N_{i}=2 \delta f_{\mathrm{c} i} / C, \quad \delta=d_{1}+d_{2}-d,
$$

where $C$ is the speed of sound. Based on the value of $N_{i}$, after calculating the sound attenuation, $\Delta L_{i}$, of the barrier from the so-called Maekawa's acoustical evaluation chart for a barrier, the frequency spectral characteristic is explicitly evaluated by:

$$
a_{i}=10^{-\triangle L_{i} / 10} \quad(i=1,2, \ldots, N) .
$$

In this case, $X_{i}(i=1,2, \ldots, N)$ in Eq. (8) is the noise intensity fluctuation existing in the $i$ th frequency band-width of the observed noise fluctuation wave at the observation point $O$ (see Fig. 1), before the barrier is constructed.

\section{EXPERIMENTAL CONSIDERATION}

3.1 Experimental Results for Sound Insulation Wall (Inside a Room)

An aluminium single-wall and an aluminium double-wall (thickness: $1.2 \mathrm{~mm}$, surface-mass: 3.22 $\mathrm{kg} / \mathrm{m}^{2}$, loss factor: $9.33 \times 10^{-3}$, double-wall air gap: $50 \mathrm{~mm}$ ) have been used. A water closet noise has been used as an input sound source in the typical living noise environment. By paying our attention to the dominant region in a frequency spectrum of the closet noise and considering the diffuse property of sound field in a room which is necessary for applying

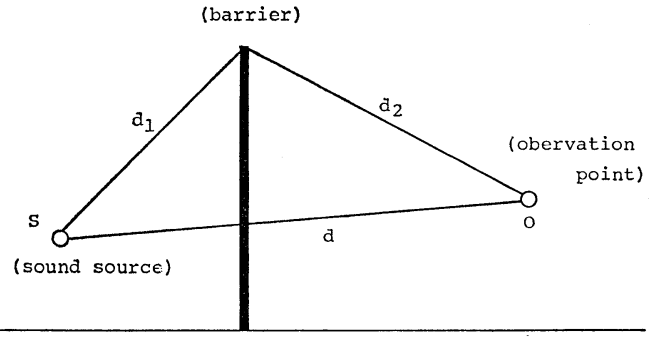

Fig. 1 Location of sound insulation barrier.

Table 1 Frequency spectral characteristic, $a_{i}$, for sound insulation wall.

\begin{tabular}{ccc}
\hline $\begin{array}{c}\text { Center frequency } \\
\text { of one-third- } \\
\text { octave band }(\mathrm{Hz})\end{array}$ & Single-wall & Double-wall \\
\hline 250 & $4.5463 \times 10^{-2}$ & $4.8489 \times 10^{-2}$ \\
315 & $3.9573 \times 10^{-2}$ & $3.0577 \times 10^{-2}$ \\
400 & $3.2391 \times 10^{-2}$ & $1.8553 \times 10^{-2}$ \\
500 & $2.4041 \times 10^{-2}$ & $1.1193 \times 10^{-2}$ \\
630 & $1.6172 \times 10^{-2}$ & $5.7115 \times 10^{-3}$ \\
800 & $1.0930 \times 10^{-2}$ & $2.8616 \times 10^{-3}$ \\
1,000 & $6.8951 \times 10^{-3}$ & $1.6089 \times 10^{-3}$ \\
\hline
\end{tabular}

the S.E.A. method, its frequency range has been adopted from $250 \mathrm{~Hz}$ to $1,000 \mathrm{~Hz}$.

The wall has been clamped between two reverberation rooms and excited via the loudspeaker. Using two sound level meters, the $\mathrm{C}$-weighted over-all noise intensity fluctuation waves, $X$ and $E$, respectively in the transmission and reception rooms have been recorded on a data recorder. The concrete values of $a_{i}$ for each sound insulation wall are shown in Table 1 by use of two S.E.A. methods. ${ }^{8)}$ In this experimental consideration, the usual one-thirdoctave band analysis has been used.

Figure 2 shows a comparison between the theoretically predicted curves by the proposed method and the experimentally sampled values obtained from the observed data $E$, for the cumulative probability distribution of the output noise intensity fluctuation, in a special case with a single-wall. The experimental results for a double-wall is shown in Fig. 3. Hereupon, let us define an expansion expression given by the only first term in Eq. (5), as the first approximation of $Q(E)$, moreover, the expression from the first term to a term with an expansion coefficient $B_{n}(n \geq 3)$ in Eq. (5), as the $(n-1)$ th approximation of $Q(E)$. 


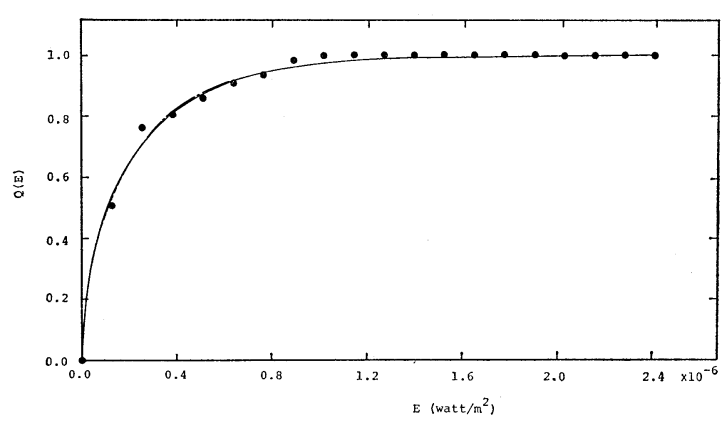

Fig. 2 Comparison between theoretically predicted curves and experimentally sampled values for cumulative noise intensity distribution (single-wall). Experimentally sampled values are marked by ( $)$ and theoretically predicted curves are shown by $\{(-)$ : the 1st approximation, (---): the 2nd approximation, (-- $)$ : the 3rd approximation\}.

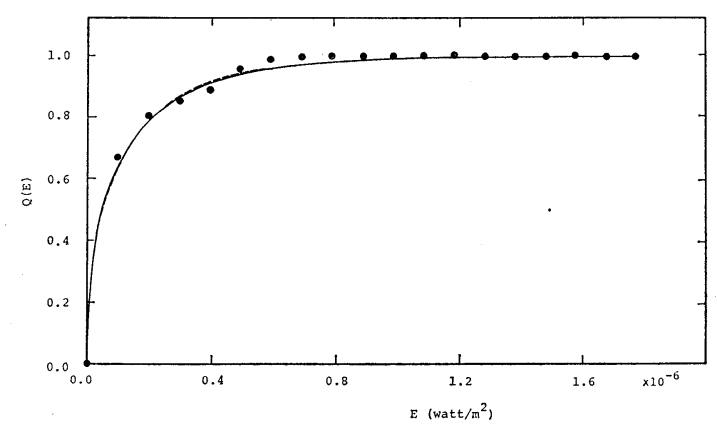

Fig. 3 Comparison between theoretically predicted curves and experimentally sampled values for cumulative noise intensity distribution (double-wall). Experimentally sampled values are marked by (0) and theoretically predicted curves are shown by $\{(-)$ : the 1 st approximation, $(----)$ : the 2 nd approximation, $(---)$ : the 3 rd approximation\}.

In each case, it is obvious that the experimental results are found to be in good agreement with the theoretically predicted curves.

\subsection{Experimental Results for Sound Insulation Bar- rier (Outside a Room)}

The experiment has been done at night $(20.00$ P.M.-03.00 A.M.) in a playground to avoid the effect of surrounding background noise. The layout
Table 2 Frequency spectral characteristic, $a_{i}$, for sound insulation barrier.

\begin{tabular}{ccc}
\hline $\begin{array}{c}\text { Center frequency } \\
\text { of octave band } \\
(\mathrm{Hz})\end{array}$ & $h=0.8(\mathrm{~m})$ & $h=1.3(\mathrm{~m})$ \\
\hline 250 & 0.07771 & 0.19498 \\
500 & 0.04023 & 0.05370 \\
1,000 & 0.01943 & 0.04897 \\
2,000 & 0.00996 & 0.02754 \\
4,000 & 0.00479 & 0.01479 \\
\hline
\end{tabular}

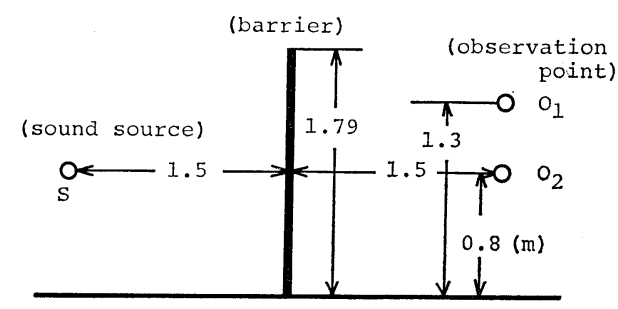

Fig. 4 Layout of sound source, barrier and two observation points.

of the sound source, the barrier and two observation points is shown in Fig. 4. The barrier made of plywood panel (height: $1.79 \mathrm{~m}$, width: $6.32 \mathrm{~m}$, thickness: $13.5 \mathrm{~mm}$ ) has been used.

As one of the typical noise environment outside a room, a road traffic noise wave has been used. Using two sound level meters, two kinds of A-weighted over-all noise intensity fluctuation waves, $X$ and $E$, before and after constructing the barrier have been recorded on a data recorder. Hereupon, for the simplification of experimental consideration, the noise intensity fluctuation wave has been observed by use of the octave band analysis.

The frequency spectral characteristic, $a_{i}$, of the barrier evaluated by using Maekawa's evaluation chart for a barrier is shown in Table 2. Hereupon, the effect of refraction from the ground has been taken into consideration.

Figure 5 shows a comparison between the theoretically predicted curves by the proposed method and the experimentally sampled values obtained from the observed data $E$, for the cumulative probability distribution of the noise intensity fluctuation, in a special case when the height of observation point is $0.8 \mathrm{~m}$ (after constructing the barrier). In another case, the height of observation point is $1.3 \mathrm{~m}$, and a 


\section{OHTA and Y. MITANI: PROBABILISTIC RESPONSE OF SOUND INSULATION SYSTEMS}

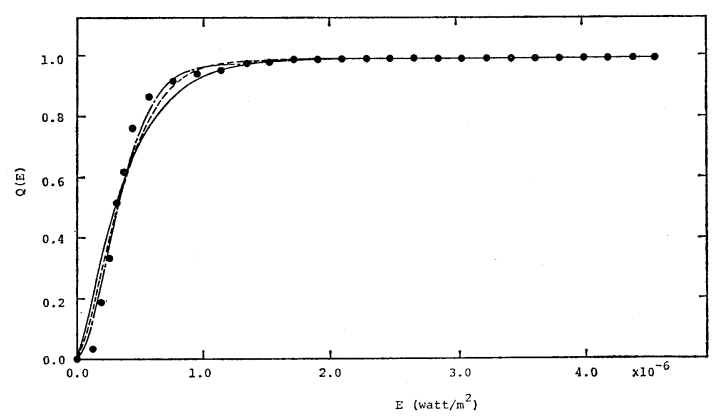

Fig. 5 Comparison between theoretically predicted curves and experimentally sampled values for cumulative noise intensity distribution (height of observation point: $0.8 \mathrm{~m}$ ). Experimentally sampled values are marked by $(\odot)$ and theoretically predicted curves are shown by $\{(-)$ : the 1st approximation, (----): the 2 nd approximation, (- - - ) : the 3rd approximation\}.

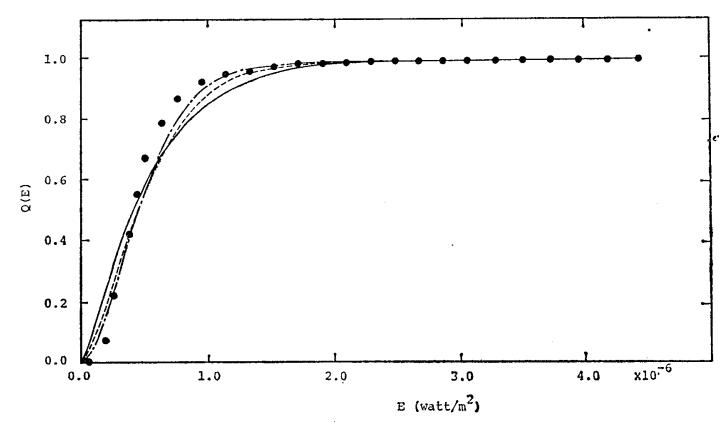

Fig. 6 Comparison between theoretically predicted curves and experimentally sampled values for cumulative noise intensity distribution (height of observation point: $1.3 \mathrm{~m}$ ). Experimentally sampled values are marked by (e) and theoretically predicted curves are shown by $\{(-)$ : the 1st approximation, (----): the 2 nd approximation, (-- $)$ : the 3rd approximation\}.

comparison between theory and experiment is shown in Fig. 6.

In each case, it is obvious that the theoretical curves are closer to the experimentally sampled values as the number of correction term increases.

\section{CONCLUSION}

In this report, a simplified probabilistic evaluation method for the output random noise fluctuation has been proposed in a unified type of probability distribution form, when a stationary random noise of general distribution type has been attenuated by the sound insulation systems such as a single-wall, a double-wall and a barrier.

Such a probabilistic evaluation research of sound insulation systems is still in an early stage of study and so its main point has been focussed on only its fundamental viewpoint. Therefore, there still remain many future problems of applying it to the other actual cases.

\section{ACKNOWLEDGMENTS}

Finally, the authors would like to thank Prof. S. Yamaguchi, Dr. K. Hatakeyama, Mr. U. Yho, Mr. N. Ohira and Mr. A. Nanba for their helpful suggestion and assistance.

\section{REFERENCES}

1) A. London, "Transmission of reverberant sound through double walls," J. Acoust. Soc. Am. 22, 270279 (1950).

2) K. A. Mulholland, H. D. Parbrook, and A. Cummings, "The transmission loss of double panels," J. Sound Vib. 6, 324-334 (1967).

3) G. Maidanik, "Response of ribbed panels to reverberant acoustic fields," J. Acoust. Soc. Am. 34, 809-826 (1962).

4) M. J. Crocker and A. J. Price, "Sound transmission using statistical energy analysis," J. Sound Vib. 9, 469-486 (1969).

5) A. J. Price and M. J. Crocker, "Sound transmission through double panels using statistical energy analysis," J. Acoust. Soc. Am. 47, 683-693 (1970).

6) C. M. Harris, Handbook of Noise Control (Mc-Graw Hill, New York, 1979), Chap. 23.

7) Nippon-Onkyozairyo-Kyōkai (Ed.), Handbook of Noise Control (Gihōdō, Tokyo, 1971), pp. 297-329 (in Japanese).

8) M. Ohta, S. Yamaguchi, and N. Nakasako, "A trial of probabilistic evaluation for the sound insulation system based on the modified method of statistical energy analysis," Acustica 56, 270-279 (1984).

9) M. Ohta and T. Koizumi, "General statistical treatment of the response of a nonlinear rectifying device to a stationary random input," IEEE Trans. Inf. Theory IT-14, 595-598 (1969).

10) H. Iwashige and M. Ohta, "A study on sound transmission loss of double-walls having several types of geometrical section by use of the improved statistical energy analysis method," J. Acoust. Soc. Jpn. 36, 447-458 (1980) (in Japanese). 